\title{
Territorios en mutación. Superación y desplazamiento de las fronteras interiores en la Patagonia chilena
}

JUAN CARLOS RODRÍGUEZ

> Universidad de Valparaíso, Valparaíso, Chile. juancarlosrodriguezt@yahoo.com

\author{
Universidad de Valparaíso \\ Facultad de Arquitectura \\ Revista Márgenes \\ Espacio Arte Sociedad \\ Territorios en mutación. Superación y desplazamiento de las \\ fronteras interiores en la Patagonia chilena \\ Diciembre 2015 Vol. $12 \mathrm{~N}^{\circ} 17$ \\ Páginas 51 a 61 \\ ISSN elec. 0719-4463 \\ ISSN imp. 0718-4034 \\ Recepción: septiembre 2015 \\ Aceptación: noviembre 2015
}

\section{RESUMEN}

En la Patagonia chilena, los territorios de Aysén y Palena han sido históricamente considerados "fronteras interiores" del Estado Nacional. Sus espacios han sido apropiados material y simbólicamente por el poblamiento espontáneo de dispersas unidades familiares vinculadas a actividades silvoagropecuarias y pesqueras tradicionales, configurándose un territorio sui generis distinto al de un país organizado con fuerte centralismo. Recientemente, tras casi un siglo desde la incorporación institucional de estos territorios, se vienen instalando modelos de desarrollo coherentes con imaginarios y fuerzas globales contrarios a los modos de vida locales, caracterizados por la agricultura familiar campesina (AFC).

En este artículo se discuten los impactos locales de la mutación de los territorios por efecto del régimen de acumulación capitalista, los que ponen en juego las estrategias tradicionales de sobrevivencia frente a modelos extractivistas y de mercantilización de la naturaleza.

PALABRAS CLAVES

Patagonia chilena, fronteras interiores, nueva ruralidad latinoamericana, modelos de desarrollo, agricultura familiar campesina

\section{Territories in mutation. Destructuration and shifts of the internal borders in Chilean Patagonia \\ ABSTRACT}

In the Chilean Patagonia, the territories of Aysén and Palena have historically been considered "internal borders" of the National State. Its spaces have been appropiate materially and symbolically by the spontaneous settlement of dispersed family units linked to traditional agriculture, forestry and fishing activities, giving place to a sui generis territory opposite to an organized country with strong centralism.

Recently, after almost a century since the institutional incorporation of these territories, have been installed development models congruent with myths and global forces opposed to local lifestyles, characterized by native family farming.

In this article are discussed the local impacts of the mutation of the territories for effect of the capitalist accumulation regime, which bring into play the traditional survival strategies against extraction and commodification of nature models.

KEYWORDS

Chilean Patagonia, internal borders, new Latin American rurality, development models, native family farming

\section{INTRODUCCIÓN}

Las poblaciones que se movían de un lado a otro siguiendo a sus ganados o desplazándose de tierras exhaustas a otras nuevas y más fértiles, se encontraban de repente impedidas de utilizar tierras que habían considerado tradicionalmente a su disposición, porque ahora estaban cercadas y rodeadas de alambre de espino por alguien que las poseía perpetua y absolutamente, aunque no las utilizara (Harvey, 2014:54)
Un excesivo economicismo en el modelo de desarrollo implementado a partir del Ilamado Consenso de Washington, ha abierto una importante posibilidad de lectura sobre la escala micro y mezo de ocurrencia de los fenómenos del crecimiento y sus manifestaciones en el territorio. Después de una docilidad obsecuente de los tecnócratas neoliberales, nadie duda que no basta el crecimiento económico per se, o el crecimiento por el crecimiento, como varia- 


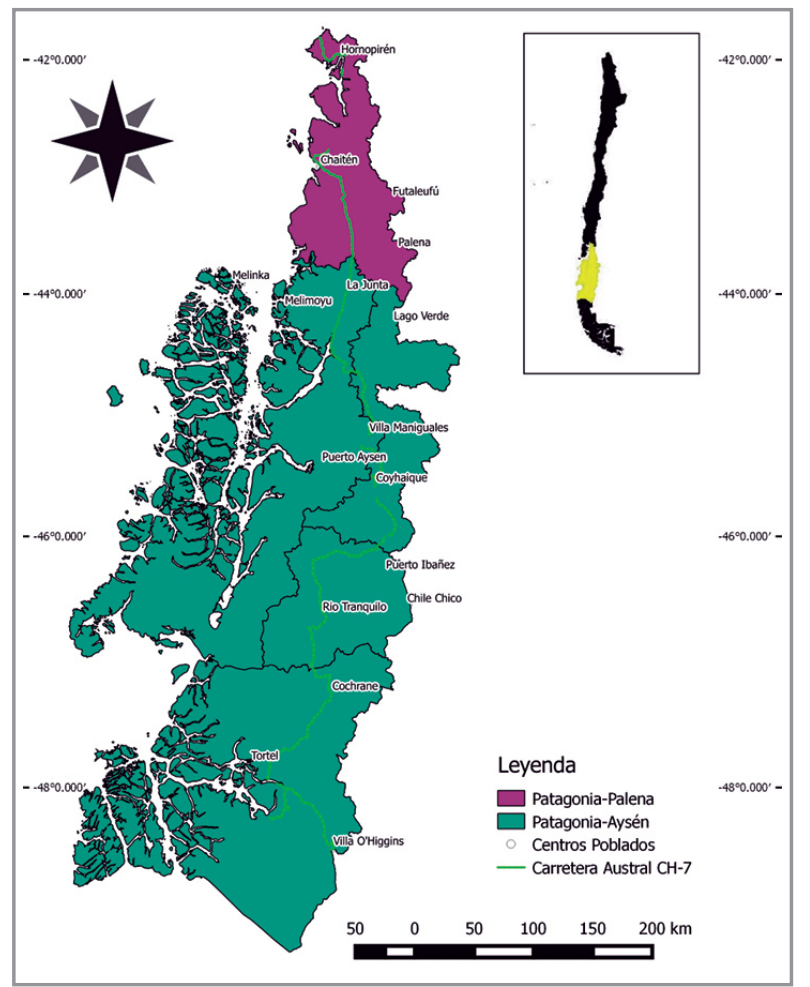

> Mapa 1. Patagonia norcentral chilena. ble indicativa del desarrollo. Asimismo, que el lenguaje de la valoración y la propia experiencia de las transformaciones se manifiestan de manera desigual en el territorio; con zonas de dinamismo, de exclusión y de sacrificio ambiental y social. Así, no podemos perder de vista la interrelación entre lo global y lo local, y lo local y global, ya que solo esta dinámica es la que permite explicar compleja y multicausalmente fenómenos localizados de emigración, concentraciones demográficas, especialización productiva, producción para la exportación, ventajas comparativas, entrada y salida de actores del mundo laboral, cambios de roles de género, diferenciaciones salariales, inversiones, nuevas tecnologías, entre otras.

De esta forma, el territorio ha entrado a jugar un papel central en el análisis de las ciencias sociales. Se les entiende pluralmente como espacios sociales construidos, cuya singularidad y sedimentación está atravesada por las estrategias de diferentes actores; individuales, colectivos e institucionales, que actúan en diferentes campos, con grados distintos de poder y que afectan los bienes comunes a partir de la identidad, la cultura y las acciones sobre la naturaleza. Esta fórmula, se ubica más allá de todo razonamiento burocrático y tecnocrático sustentado en fraccionamientos geográficos o jurídicos estableciendo claramente la diferencia entre el territorio y el mapa, ya que son los actores locales los que hacen uso de ecosistemas particulares y definen las extensiones de los mismos, como lo ha demostrado la antropología en sus estudios más clásicos.

Lo que queremos presentar en este artículo, son los cambios obrados en los territorios rurales en una condición particular: en lo que son las fronteras interiores de Chile, específicamente en la Patagonia - Palena y Patagonia - Aysén (Mapa № 1).

\section{FRONTERAS INTERIORES}

Las fronteras interiores, término acuñado especialmente en el mundo militar (Masalleras \& Ortega, 2012), definen una territorialidad sui generis: baja densidad demográfica, problemas de conectividad, ausencia de servicios especializados, históricamente con pocas variantes y con una cultura que está lejos de la identificación de la simbología nacional. Se trata de una condición de soberanía precaria vulnerable y que se pone al límite de la integración nacional, ya que no hay una conexión efectiva con el gobierno central y/o regional, destacando las dificultades para desarrollar actividades económicas y sociales por la distancia existente con la administración central del país, en un régimen de tipo unitario y por sobre todo centralista. En este sentido, lo que se vive y experimenta en términos locales está lejos de la imaginación de tipo nacional al modo de Anderson (2006) y lejos también de imaginar o pensar localmente y desde el día a día los problemas país, ya que constituyen entidades culturales, demográficas y territoriales independientes (Masalleras \& Ortega, 2012:138), que desnudan el fracaso de la regionalización.

Como antecedentes históricos que pueden explicar sucintamente por qué la Patagonia, al sur de paralelo 42, cuenta con una densidad demográfica que no supera los 0,9 habitantes por $\mathrm{km}^{2}$, proponemos las siguientes:

1. Un territorio estigmatizado temprana y negativamente a través navegantes y figuras canónicas como Darwin, e historiadores como Barros Arana, cuyos discursos predominaron hasta fines del siglo XX.

2. Una base de actividades predatorias rápidas y altamente lucrativas en la zona más austral, como fue el comercio de 
pieles de avestruz, lobos marinos y guanacos en la parte más austral; junto a la extracción desmedida de recursos forestales como el alerce (Fitzroya cupressoides) y el ciprés de las guaitecas (Pilgerodendron uviferum), exportados para durmientes de ferrocarril a Perú, elaboración de tejuelas y embarcaciones, y utilizados como moneda.

3. La forma de adjudicación de la tierra favoreció el acaparamiento de grandes extensiones en manos de particulares y pocas empresas, con administración a distancia, desde sus casas matrices en ciudades centrales.

4. El privilegio de trabajadores solteros por sobre familias para el trabajo ganadero y forestal.

5. La ausencia de planificación de asentamientos urbanos que marcaran la presencia del Estado y su institucionalidad.

6. Una actividad monoproductora como la ovejería lanar, que en la estepa sobrepasó la capacidad de carga de los ecosistemas.

Lo señalado constituye la carga histórica del territorio y de las relaciones, que se complementan a través de la fijación de límites fronterizos que buscaron argentinizar y chilenizar la población (Baeza, 2009), aunque la práctica de la trashumancia fue una constante anterior a la formación de ambos estados, y en la actualidad mantiene una vigencia permanente a través de relaciones políticas y consanguíneas, articuladas además comercialmente y a través de servicios.

En general, la ruralidad de este amplio territorio de bajísima demografía y alta dispersión, se configura a través de una estructura de propiedad peculiar. Por una parte, grandes extensiones de tierra controladas por unidades domésticas que las explotan con sistemas agropecuarios extensivos de baja productividad. Por otra, es posible asociar estas unidades a la Agricultura Familiar Campesina (AFC), concepto que incluye una diversidad de agentes y actores como campesinos, pequeños y medianos productores, medieros, productores sin tierra, parceleros, entre otros, que realizan distintas actividades agrícolas, forestales, ganaderas o pesqueras (FAO, 2014), que más que ser conceptualizadas como un modo de organizar la producción, constituyen modos de vida cuyo fin es la reproducción social de la familia en condiciones dignas (Craviotti, 2014).

El trabajo de campo ayuda a comprender que las condicionantes geográficas, económicas y sociales no permiten una alta productividad de los suelos así como de comercialización de productos locales. Que un predio de 200 hectáreas no signifique mayor riqueza e incluso requiera extensivamente de otros espacios estivales para el forrajeo, como son las veranadas en el caso de la ganadería; o, que pese a la abundancia de recursos marítimos, la fijación estatal de cuotas de extracción haga en ciertos casos insostenible la reproducción de la actividad de la pesca artesanal en localidades del litoral.

La importancia de las AFC, tanto en los archipiélagos y fiordos del litoral como en las distintas ecoregiones continentales ${ }^{1}$, es que corresponden al segmento poblacional de mayor arraigo y tiempo de residencia en la zona (Villagrán et al, 1997), y que sus complejos sistemas económicos-tradicionales que articulan al mismo tiempo estrategias de supervivencia y acumulación estacional, han sido modelos socio-productivos que configuran la apropiación histórica del territorio y explican el éxito del poblamiento espontáneo frente a los programas de colonización estatal, en un proceso continuo de aprendizaje y adaptación en el que se forjan distintas formas de relación con el medioambiente.

La especial ruralidad de la región de Aysén y la provincia de Palena también se incluye dentro de los procesos latinoamericanos

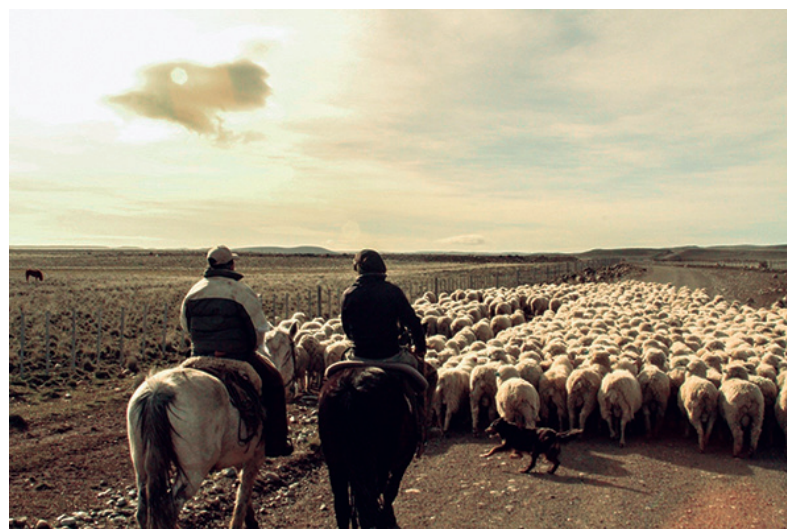

Figura 1. Ovejeros en Ñirehuao, comuna de Coyhaique. Fuente: Patricio Blanche, 2015 
de reorganización económica y territorial llamada Nueva Ruralidad (Barkin, 2004; Bonnal, 2004; Carton de Grammont, 1999; Carton de Grammont y Martínez, 2009); un proceso considerado heterogéneo en sus manifestaciones, de acuerdo a los distintos contextos políticos-nacionales y económicos donde operan (Llambí en Gómez, 2000). En general, se ha observado el proceso como un tránsito de lo agrario a lo agroindustrial, lo que involucra una serie de alteraciones en la vida de comunidades rurales, que pueden incluir el desenmarañamiento del tejido social y/o la reinvención de formas sociales locales (Salas, Rivermar y Velasco, 2011).

Como veremos, la Patagonia chilena presenta matices peculiares de alteración que no implican necesariamente el tránsito a lo agroindustrial como sí ocurre en la zona central del país. La Patagonia se encuentra en un proceso creciente de disputa por control territorial similar a lo documentado por Margarita Serje (2011) en aquellas consideradas Tierras Salvajes o Tierras de Nadie en Colombia, donde se ha planteado si el ausentismo estatal no será en realidad una forma de gobierno para constituir periferias disponibles a la penetración del capital. 0, como sostiene Harvey, que la propiedad privada establece un derecho de pertenencia exclusiva sobre un objeto o un proceso, ya esté siendo utilizado activamente o no...[porque] El derecho de propiedad privada confiere la posibilidad de vender (enajenar) lo que se posee. Se crea así una diferencia entre lo que se llaman derechos de usufructo (que corresponden al uso activo) y derechos de propiedad exclusiva permanente (Harvey, 2014:53-54).

Nuestra tesis, por una parte, señala que toda mutación en la ruralidad pone en juego las estrategias tradicionales de sobrevivencia de las AFC (o, la memoria si se quiere), de poblaciones invisibilizadas dentro de un país políticamente unitario; por otra, que en este territorio se están jugando dos versiones del capitalismo: el que tiende a la infinitud de la explotación de los recursos en un planeta finito, y el conservacionista verde, que renta con los ecosistemas a través de su preservación, con fines filantrópicos de contención, re reforestación para vender bonos de carbono y el desarrollo de circuitos de turismo únicos de experiencia, no masivo y anónimo.

La mutación del territorio rural patagónico en cualquiera de las fórmulas, por separado o combinadas, pone a prueba el capital social de las comunidades. Entonces, es la cultura, la experiencia, la cosmovisión y la autonomía lo que se reconfigura a través de los nuevos controles de la tierra. Así, estas unidades rurales aisladas, quedan atrapadas por las redes de las propias contradicciones que sufre el capitalismo en los espacios y territorios interiores: el depredador y el conservacionista. De este modo, no nos referimos a la contradicción entre capital y trabajo, sino a la contradicción entre presión por los recursos de manera ilimitada y recursos finitos cuando la tierra se convierte en suelo.

\section{ESTRATEGIAS DE DESARROLLO EN CONFLICTO: EL NUEVO CONTEXTO PATAGÓNICO}

En Chile, al término de la dictadura militar en 1989, se inicia un proceso de nueva territorialización en los espacios australes, marcado por la liberalización económica (Rodríguez et al, 2012; Núñez et al., 2014) que ha dejado en evidencia una cuestión histórica: 1. el naufragio de las propuestas de colonización, avalado por ausencia de políticas efectivas por parte del Estado de consolidar su institucionalidad y presencia; 2 . incapacidad para producir sinergias regionales virtuosas e incentivos para generar arraigos en la población; y, 3. extrema dependencia de factores exógenos para producir o generar cambios. Creemos, esto ha mantenido la condición de lo que se conoce como "frontera interior".

La consolidación de las políticas neoliberales en la Patagonia, se expresa en el asentamiento incompatible de fórmulas de desarrollo que presionan fuertemente la ruralidad tradicional: a. el extractivismo, que demanda intensa explotación y transformación del entorno natural y social, también conocido como Principio Potosí (Machado, 2014 a y b), la mercantilización de la naturaleza asociada a actividades "blandas" y discursos dirigidos al desarrollo sustentable, asentados en la conservación de la naturaleza con rentabilidad económica (Núñez et al, 2014), o a la eliminación misma de la idea de desarrollo y progreso. La incorporación de ambos modelos, forma parte de las estrategias de desarrollo que los gobiernos regionales ejecutan, pues diagnosticando la situación de frontera interior que amenaza la integralidad nacional, se han generado sucesivos planes de intervención durante por lo menos dos décadas (Hartmann, 2009), visualizando el problema austral entre demografía, extensión territorial y productividad, como parte de un deficitario ordenamiento territorial. Sin embargo, dado el trasfondo de liberalización económica como paradigma irrevocable (o incuestionable para muchos), no sólo se ha permitido el asentamiento de modelos de comodificación de la naturaleza (Romero, 2009), sino que además de enfoques desvinculados de los modos de vida locales, e incluso de los gobiernos locales, conformando un territorio conflictivo, en disputa, o un campo de posibles debido a la valorización diferenciada de los recursos y el territorio (Rodríguez et al., 2012).

\section{El modelo extractivista}

Apoyado en la contradicción capital/naturaleza (Galafassi, 2012; Harvey, 2014), es capaz de comodificar recursos y territorios patagónicos por su valor de cambio y transformar en commodities los elementos medioambientales a los que el capital tiene acceso. Esto incluye la privatización, mercantilización, monetización y comercialización del agua, el bosque, los minerales, las zonas costeras. Así, los atributos de la bio-región austral pueden ser valorados como recursos exportables, mediados por la sofisticación científico-tecnológica alcanzada (Delgado, 2013).

En particular, el extractivismo se ha afianzado en la zona de modo divergente, según los ejes de poblamiento oriental y occidental que estructuran la Patagonia norcentral chilena (Villagrán et al, 1997). En el continente, por ejemplo, los recursos tradicionalmente utilizados como los madereros, hídricos y praderas son amenazados progresivamente por proyectos energético-mineros que operan deslocalizadamente, solicitando el uso exclusivo de las cuencas hídricas y arroyos que han permitido la supervivencia de las AFC durante un siglo. Por cierto, las AFC no se enteran hasta que asesoradas por el Instituto de Desarrollo Agropecuario (INDAP), viajan a las capitales regionales para realizar algún proyecto de riego, viéndose imposibilitados para su habilitación por ser aguas solicitadas por empresas transnacionales como ENDESA, para fines hidroeléctricos ${ }^{2}$. Existen otros casos que van adquiriendo mayor relevancia como son las actividades mineras, que en la localidad de Mañihuales (XI Región) ya han costado la vida de los primeros animales, posiblemente por contaminación de acuíferos y pastizales ${ }^{3}$, aunque la mayor polémica se ha desatado luego de que siete pobladores en la misma zona arrojaran positivo en exámenes para verificar altos niveles de metales pesados en la sangre ${ }^{4}$. Sumado a lo anterior, puede sostenerse que el mayor impacto se ubica en la intensidad especulativa y adquisitiva que han generado los terrenos, por la multiplicación de so- 
licitudes de uso en el último tiempo, como parte de la denominada fiebre del oro de Aysén y Palena, controlando ya 635 mil hectáreas ${ }^{5}$.

La exploración minera y las solicitudes de explotación han introducido alteraciones y grandes expectativas en la población rural, que por un lado observa estas incursiones como la posibilidad real de que crezca el mercado laboral, frenando el proceso migratorio de jóvenes; por otro, también observando una amenaza creciente a su forma de vida, pues reconocen que de ser solicitado el subsuelo de sus propios predios, serán expulsados a una vida urbana con la que no se identifican. Frente esto, señalaba un habitante del Valle El Espolón ${ }^{6}$, que: le incomodaba e intranquilizaba el sólo hecho de ver tanta gente pasar frente a la ventana, cuando viajaba al poblado de Futaleufú, el que tiene menos de dos mil habitantes. Del mismo modo, el conflicto desencadenado por la irrupción de grandes proyectos energéticos y/o mineros, ha desatado movimientos de resistencia y defensa del medio ambiente antes de su ejecución, que incluyen procesos de internacionalización, como ocurrió con el emblemático Proyecto Hidroaysén (Reyes y Rodríguez, 2015), dividiendo a la población en torno a si es más ventajoso el extractivismo o el desarrollo de otra actividad como el turismo, carente también de sustento histórico en las AFC tradicionales (Segura y Bourlon, 2011).

Sin embargo, es en el litoral donde el modelo extractivista se ha consolidado definitivamente, provocando el mayor impacto social y económico sobre la ruralidad. Se trata de la violenta y súbita radicación de dos tipos de industrias: pesquera y acuícola. La primera, desde 1985 establece uno de los últimos procesos de poblamiento del litoral (Ramírez, 1998), con familias de pescadores artesanales atraídos por repentinas sobre-demandas de la industria exportadora, conocidas como fiebres sobre recursos marítimos que se encuentran en los canales: loco, pelillo, merluza, entre otros. Hoy, es una industria pesquera estabilizada en la captura demersal (merluza austral y el congrio), insertándose de manera crecientemente conflictiva con las localidades, ya que enfrenta a antiguos y recién llegados, a los por cuenta propia y los asalariados. En particular, se instala un conflicto por las ventajas propias de la economía a escala de la industria pesquera, favorecida con la mayor cuota global otorgada por la Subsecretaría de Pesca, al mismo tiempo que disminuye para el subsector artesanal. También, porque resulta una competencia extremadamente desigual para los artesanales si se considera que son las mismas empresas las compradoras de sus desembarques. Con cinco naves industriales en el año 2014, el sector industrial superó más de 50 veces el desembarque de más de 3.000 pescadores artesanales patagónicos.

La desigualdad resultante de esta disputa, se intensifica con el sector acuícola, principal actividad económica en el litoral patagónico, ya que genera retornos de 4.200 millones de dólares con la producción de 900.000 toneladas, como fue en el año 2014. Son 719 concesiones distribuidas en 30 empresas; y el $94 \%$ de ellas opera en el mar? ${ }^{7}$. Sin ir más lejos, con esta actividad se han concretado los principales temores locales manifestados por los megaproyectos continentales no ejecutados como Hidroaysén, pues la acuicultura requiere entre 25.000 a 47.000 trabajadores, lo que ya supera la población total de la Provincia de Aysén, en cuyas pequeñas localidades del litoral se ubican.

Nuestros registros demuestran la fragilización y fragmentación socio-espacial, correspondiente al avance longitudinal de la industria pesquera y acuícola en las localidades rurales del litoral, así como un avance de la contaminación de las aguas y el litoral por sus

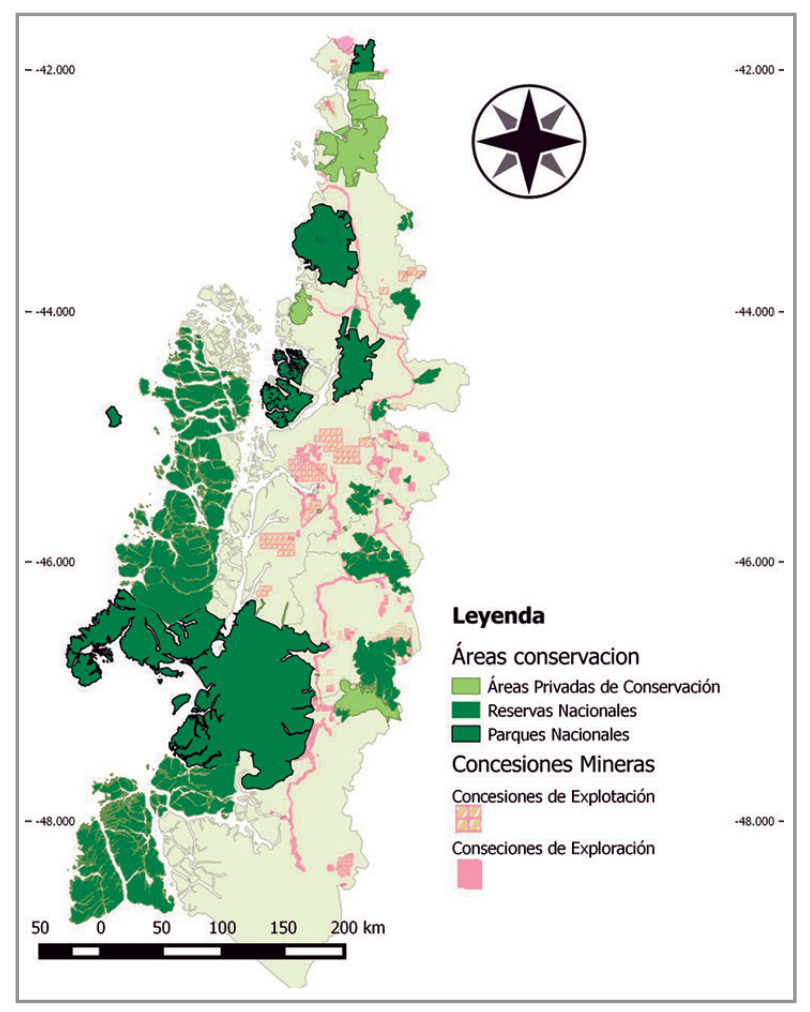

> Mapa 2. Nuevas territorialidades en Aysén y Palena. 


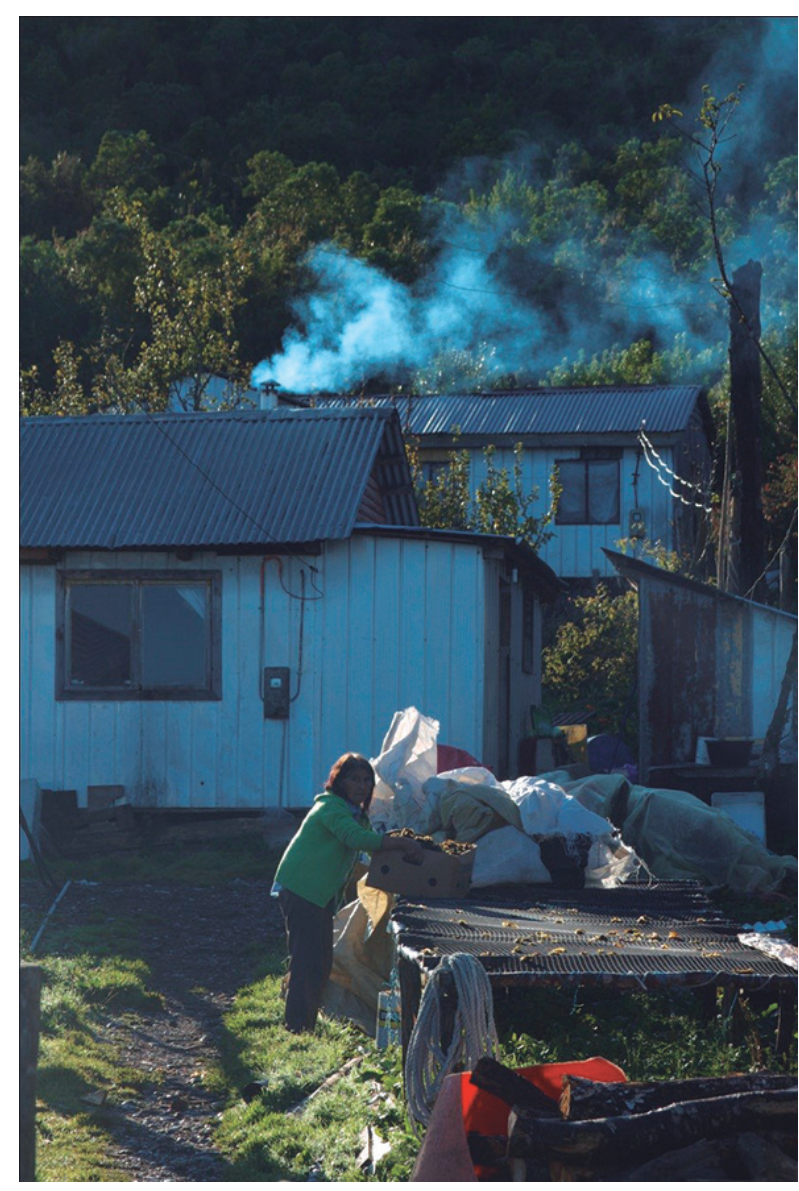

procesos. En especial, se observó la segmentación social cada vez más acelerada en localidades que antes se caracterizaban por su homogeneidad y por los fuertes vínculos comunitarios en pequeñas poblaciones nucleares. Los buzos tradicionales, por ejemplo, perciben una presión cada vez mayor en su actividad, potenciando su incorporación como empleados de alguna de las empresas, lo que significa para muchos de ellos no sólo precarizar sus vidas con sueldos inferiores a los que percibían, sino también en la pérdida de la autonomía, que ha sido una de las características más resistentes de la ruralidad tradicional.

Así, el Plan de Ordenamiento Territorial de la Región de Aysén (2011) indicaba que el PIB sectorial de Pesca y Acuicultura era el de mayor crecimiento en el PIB regional, al mismo tiempo que disminuía el aporte artesanal, desde un 42\% en 1990 a 18\% el 2001. En otro sector importante, la minería en el período entre 19852006 experimentó un crecimiento de 14,8\% anual, mientras que el sector agropecuario-silvícola disminuía a un 0,5\% anual.

\section{Mercantilización de la Naturaleza}

Estudiada académicamente como un nuevo proceso de colonización en la Patagonia (Rodríguez et al, 2012; Núñez et al, 2014), se asocia la mercantilización de la naturaleza a una forma renovada de capitalismo post-industrial, que redefine y reconfigura los espacios rurales con fines no productivos en el sentido clásico. A diferencia del modelo extractivista, propone la obtención de una rentabilidad monetaria o no monetaria a través de la conservación de ecosistemas, y en otros aprovechando lo que contienen los mismos, dando énfasis en la singularidad de los remotos y aislados paisajes australes para vender experiencias verdes o ser solidarios con el planeta. Por ejemplo, reconocemos la venta de bonos de carbono, restauración de praderas y ecosistemas expulsando a la fauna exógena, generar corredores -mediante compra de tierrasque se unan con reservas y parques nacionales para la reproducción de la fauna endémica.

En el contexto de liberalización económica, se desarrollan distintas conceptualizaciones y producciones de valor material y simbólico para la acumulación de capital en la Patagonia, mercantilizando paisajes y en algunos casos culturas locales consideradas singulares. En general, hemos registrado la incorporación de actividades no tradicionales asociadas a este modelo, que ejercen presiones sobre la vida tradicional de las AFC. Entre otras, se observa la consolidación de eco-corretajes, el auge de parques de conservación privados, la expansión de la industria turística de intereses especiales, la venta de bonos de carbono, e incluso, un nuevo proceso migratorio de amenidad (Moss, 2006). Todos estas modalidades constituyen presiones verticales, a través de fuerzas globales (Santos, 2000) y prescindiendo de la vida local.

Se trata de un proceso de re-territorialización que se ha concentrado principalmente en áreas continentales, facilitado por la apertura de la Carretera Austral, la que desde la década de 1980 permite la exploración de los paisajes a actores foráneos motivados por intereses diversos. Por ejemplo, el río Futaleufú en la Provincia de Palena, hoy reconocido mundialmente como uno de los favoritos para la práctica de kayak y rafting, fue puesto en valor entre 19841985 por Mark Allen, Phil DeReimer, Lars Holbek, Eric Magneson, y posteriormente por el miembro del equipo olímpico norteamericano, Chris Spelius, quienes transmitieron el rumor de aguas turbulentas calificadas como de otra liga (Langman \& Moore, 2013).

Figura 2. Pobladora de Puerto Aguirre secando cholgas (Aulacomya atra), en el litoral aisenino. Fuente: Patricio Blanche, 2015.

Actualmente, Spelius es uno de los empresarios turísticos más 
sobresalientes en Futaleufú, aunque reconoce con algo de nostalgia que las transformaciones desencadenadas por su esfuerzo de visualizar el río a nivel internacional, han sido de alto impacto y no siempre positivas (ibíd.).

La mercantilización de la naturaleza a través de la revalorización simbólica de paisajes, es consecuencia de la histórica desvinculación de la Patagonia con los proyectos nacionales de desarrollo que llegaron hasta el Chile central. En esta puesta en valor, la intención es articular los espacios aislados con la sociedad global. En este sentido, se activa a través del control de la tierra y sus ecosistemas un circuito desterritorializado de goce de los atributos. Sin embargo, paralelamente, resulta intrigante que las poblaciones rurales patagónicas no se articulen significativamente a los mercados globales como lo han sido sus paisajes, prueba de ello es la deficitaria oferta de servicios turísticos locales frente a la multiplicación reciente de grandes infraestructuras tipo lodges, disponibles para un circuito internacional de buscadores de elementos de distinción. Se trata de negocios hiperexclusivos que se han triplicado y concentrado en la Región de Aysén en las últimas décadas, con 65 establecimientos frente a 13 desarrollados en Magallanes al año $2011^{8}$. Mientras, el turismo rural ofrecido por las AFC, no articula a más de 40 hogares familiares distribuidos desde la Provincia de Palena hasta la Provincia del Capitán Prat, los que elegidos y convertidos en adjudicatarios de fondos públicos, solo han logrado mejorar sus viviendas pero no su condición de vida.

Entonces, la Patagonia se escinde en estos dos circuitos de apreciación y disfrute de sus ecosistemas y belleza escénica. Sin embargo, la expansión de la Industria del Ocio desestabiliza la vida aislada rural, con una acelerada consolidación de lógicas duales. Una extensa temporada baja, que da continuidad del aislamiento y la soledad principalmente en invierno; y, una temporada alta, con un aumento exponencial en la densidad demográfica en el verano. Sólo entre diciembre 2014 y marzo 2015, en la Región de Aysén -con menos de 100.000 habitantes- ingresaron 85.979 turistas, impactando severamente en localidades como Puerto Tranquilo, un pueblo rural de 400 habitantes aproximadamente que durante el verano aumenta su población a más de 5.000 personas, con turistas atraída/os por las Capillas de Mármol y el Glaciar Exploradores. Además, el circuito de altos ingresos tiene una logística desconocida e invisible para la mayoría de la población rural, imperceptible en su despliegue y un impacto cero en sus vidas.

Es importante señalar, que hasta ahora no existen estudios conocidos sobre la capacidad de carga de los poblados y los ecosistemas. $Y$ que si bien se multiplica el empleo temporal, sigue siendo un aspecto curioso la extrema dependencia alimentaria de la región.

La actividad turística, además se ha convertido en un importante motor para nuevas apropiaciones y lecturas territoriales, puesto que muchos de los nuevos actores y residentes en la zona han Ilegado inicialmente como turistas. Entre otros, el empresario Warren Adams llega a la Patagonia a través de un recorrido por el mundo, luego de vender una red social virtual adquirida por Amazon en US\$ 100 millones en 1999. A pesar de los sorprendentes paisajes que conoce en este viaje, fue la Patagonia el territorio que más lo cautivó con la idea de hacer algún tipo de negocio. Actualmente, desarrolla un proyecto que busca vencer una de las grandes contradicciones del capitalismo, Capital y Naturaleza (Harvey, 2014), mediante la empresa Patagonia Sur, dedicada al eco-corretaje y la venta de bonos de carbono tanto en la Provincia de Palena como
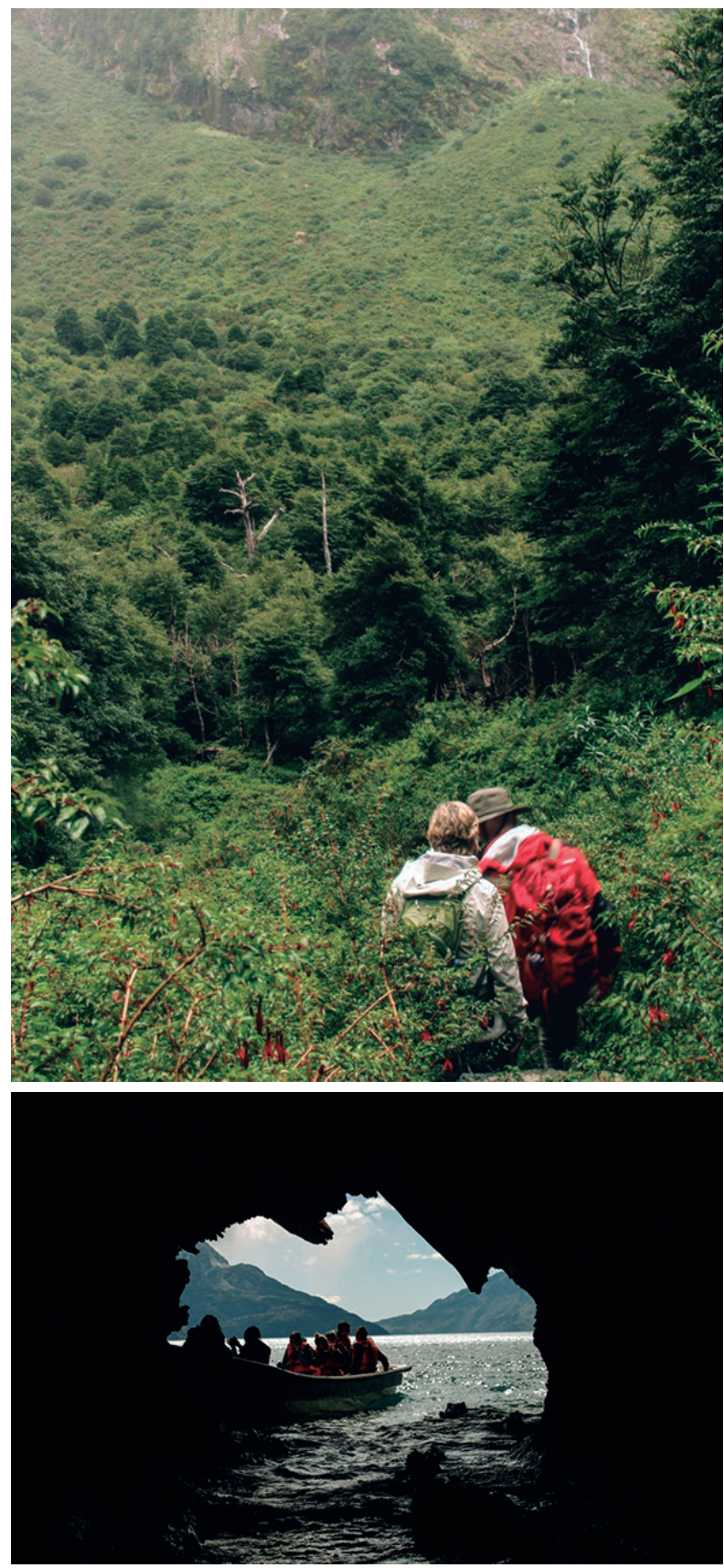

> Figura 3. Expedición en Bosque Encantado, Parque Nacional Queulat, Comuna de Cisnes. Fuente: Patricio Blanche, 2015.

Figura 4. Turistas en Capillas de Mármol, Puerto Río Tranquilo, Comuna de Río Ibáñez. Fuente: Patricio Blanche, 2015 
en la Región de Aysén. El proyecto permite generar utilidades mediante la compra de grandes extensiones de tierra, originalmente agropecuarias, para levantar Reservas Ecológicas Privadas, y aprovechando la legalidad chilena, utilizar hasta el $20 \%$ de la superficie total para la venta de parcelas. De esta manera, la empresa ha logrado su objetivo que es proteger y desarrollar propiedades escénicamente extraordinarias y ecológicamente valiosas en la $\mathrm{Pa}$ tagonia chilena.

Patagonia Sur también es una de las empresas pioneras en Chile de venta de bonos de carbono, donde nuevamente se desestabiliza la contradicción capital/naturaleza, pues mediante dos procesos simultáneos de conversión de la tierra en suelo y objeto de especulación, junto a la reforestación nativa para la captura de $\mathrm{CO}_{2}$, introducen una nueva lógica en que la conservación ecológica puede generar importantes utilidades. En particular, Patagonia Sur tiene 90.402 árboles nativos plantados, una captura de 45.201 toneladas de $\mathrm{CO}_{2}$, y ha creado 7 bosques. Entre sus clientes se encuentran universidades norteamericanas y Land Rover ${ }^{9}$.

Si bien Patagonia Sur es un negocio exclusivo, el mercado inmobiliario y el auge de reservas privadas de conservación son dos modelos complementarios que han logrado consolidarse en la Patagonia, no sin generar conflictos locales con las distintas AFC que hacían usufructo de éstos espacios para la reproducción de su ganado y el bosque como fuente de recursos. En particular, este control extensivo es importante porque la disponibilidad de áreas productivas agropecuarias no supera el $20 \%$ de la superficie total, mientras que las Áreas Silvestres Protegidas por el Estado (SNASPE) corresponden al 50\% de la Región de Aysén y 40\% en la Provincia de Palena, lo que puede ampliarse aún más si consideramos la extensión de los recientes predios de conservación ecológica (Mapa 2), que también ejercen presión sobre las AFC dependiendo del eje en que se encuentren:

- $\quad$ En el continente, las AFC requieren de veranadas - espacios estivales de talaje extrapredial para evitar el problema forrajero que adolecen por la baja productividad de sus suelos-, al mismo tiempo que fundaciones u ONG's solicitan los mismos espacios para conservación ecológica, como ocurre por ejemplo en el proyecto de conservación de huemules de la Fundación Las Margaritas en el predio fiscal de 12.000 hectáreas llamado Río Azul. Éste es utilizado permanentemente como veranada por las AFC en Villa O'Higgins, donde se ha cuestionado la mayor importancia atribuida al heráldico huemul frente a las prácticas ganaderas de los pobladores locales. Se argumenta que sus vacas afectarían al animal en peligro de extinción ${ }^{10}$. Esta problemática se repite en otros espacios de la Patagonia, desde el Parque Pumalín en la Provincia de Palena al Parque Patagonia en la ex estancia Chacabuco en la XI Región, propiedad del recientemente fallecido ambientalista Douglas Tompkins.

- En el litoral, los casos son menos frecuentes, sin embargo sus extensiones son igualmente abrumadoras. Por ejemplo, en Melimoyu, tras las deficitarias condiciones que mantienen a unas 16 familias originarias de la colonización del lugar, hoy se emplazan distintos proyectos privados de reservas ecológicas como las de la Fundación The Conservation Land Trust (la misma del Parque Pumalín y Parque Patagonia), Patagonia Sur y la Fundación MERI. Pero hay casos de posibles conflictos, como en la isla Traiguén, la que recientemente fue vendida por el Ejército de Chile a Inversiones CIFCO S.A. por \$37.000 la hectárea, solicitando el desalojo inmediato de la población que en ella vive, en particular la Comunidad Huilliche Nahuelquín-Delgado, así como de otras familias que habitan hace más de 60 años en la isla. Por su parte, Inversiones CIFCO ha entregado el territorio en arriendo a la Fundación EcoScience para la conservación de la rana de Darwin, indicando no haber tenido conocimiento de la comunidad indígena sino posterior a la compra ${ }^{11}$.

Estos casos se repiten en toda la zona austral, en un proceso de producción y consumo de lugares (Dimitriu, 2002), entendiendo que a diferencia de los modelos extractivistas que tienden a articular la Patagonia como mercancía por sus recursos disponibles, la mercantilización de la naturaleza opera con la idea de Patagonia como marketing, una geomarca (Rodríguez, Medina y Reyes, 2014). Es decir, se vende el paisaje y la experiencia de vivir la naturaleza, lo que creemos gatilla umbrales mayores de autoexpresión y sensibilidad respecto de la conservación. Lo interesante, es el cambio y las preguntas que surgen como posible transformación de sí. Una cuestión central es ¿cómo se organiza la concreción de nuestra experiencia y en función de qué nuevos objetivos de vida? ¿Qué cosas y dimensiones se fortalecen respecto de la naturaleza y la vida una vez que se tiene conciencia de la experiencia de la Patagonia y del fin como vida imaginada?

\section{COMENTARIOS FINALES}

La idea de una Nueva Ruralidad en Latinoamérica, en la Patagonia toma la forma de procesos que comprometen diversas variables, lógicas y relaciones de poder. Los procesos de re-territorialización asociados a un modelo neoliberal extremo y puro, nos muestran la articulación de espacios locales-aislados con circuitos globales, pero en la mayoría de los casos prescindiendo del vínculo con las poblaciones locales. La privatización y fragmentación socioespacial desencadenadas por procesos globales devienen crecientemente en fuerzas de agresión y expulsión de formas locales de agricultura familiar campesina (AFC), con posibilidades restringidas para mover el ganado en lo que se consideró "tierras libres" durante gran parte del siglo XX, lo mismo para las unidades pesqueras artesanales en los canales patagónicos. Pareciera que las unidades familiares campesinas se convierten en víctimas y victimarias simultáneamente, con pocas posibilidades de convertirse en verdes o en ecologistas, en un sistema global donde circulan y se valoran cada vez más las actitudes de defensa medioambiental. Se imponen nuevas lecturas sobre el territorio, con otras escalas imaginarias, con prácticas exclusivas, anónimas e invisibilizadas como el turismo de altos ingresos, y economías de escalas mayores como la industria acuícola frente a la pesca artesanal, incompatibles con las tradicionales formas productivas agropecuarias y de pesca. Las empresas, fundaciones y ONGs compiten por los mismos espacios, que desde el punto de vista de la memoria productiva y ecosistémica están transformados en territorios, integrados y naturalizados como propios por parte de las comunidades.

Se observa también el establecimiento de nuevas relaciones entre poblaciones humanas y el medioambiente, en condiciones favorecidas por el déficit de infraestructuras y acceso a servicios sociales que permiten un arraigo digno, en zonas jurídicamente chilenas y de colonización espontánea de territorios fronterizos. Hoy, podemos decir, que diversos actores e imaginarios globales sustentados en mayor capital económico, social, cultural y simbólico, y con 
desarrollos tecnológicos de punta (aviones, helicópteros), pueden controlar espacios des-localizadamente, mitigando las condiciones de aislamiento sufridas por antiguos colonos, presionando crecientemente a poblaciones locales para la venta de sus predios o para transformarlos en nuevos sujetos rurales. Así, hemos registrado los desplazamientos de las culturas del trabajo con la presencia ex campesinos ofreciendo su fuerza de trabajo como asalariados o utilizados como carpinteros para la construcción de infraestructuras tipo lodges. Se trata de empleos flexibles y estacionarios; mientras, en paralelo, la vida tradicional trata de sobrevivir como ha sido desde la ocupación misma de los territorios desalambrados. Esta vez, la diferencia es que resulta cada vez más difícil sostener que la autonomía constituye un valor.

El espacio y el territorio están mutando y cambiando las relaciones de propiedad, pero existe un temor latente: que en el país más neoliberal del mundo, este territorio, una de las últimas reservas de vida del planeta, sea vaciado y sacrificado material y simbólicamente como ha acontecido a lo largo del resto de Chile. Y, en este sentido, las iniciativas privadas - como fundaciones conservacionistas- han jugado un papel central: son, junto a algunos movimientos sociales, la voz nacional e internacional que se opone a la depredación del capitalismo extractivista y ante la ausencia de una política de Estado sostenible y sustentable, frente a los ecosistemas vulnerables.

\section{BIBLIOGRAFÍA}

ANDERSON, B. (2006) Las comunidades imaginadas. Reflexiones sobre el origen y la difusión del nacionalismo. México, D. F.: FCE.

ARENAS, F., SALAZAR, A. y NÚÑEZ, A. editores (2011) “El aislamiento geográfico: ¿problema u oportunidad? Experiencias, interpretaciones y políticas públicas", Serie Geolibros $N^{\circ} 15$, Santiago de Chile: Instituto de Geografía, Pontificia Universidad Católica de Chile.

BAEZA, B. (2009) "Fronteras e identidades en Patagonia central (1885-2007)", Rosario: Prohistoria Ediciones.

BARKIN, D. (2004) “La nueva ruralidad y la globalización”, En: Edelmira Pérez, Adelaida Farah y Hubert Carton (Comps.), La nueva ruralidad en América Latina, Tomo II, Bogotá: Pontificia Universidad Javeriana, pp. 21-40.

BíOBío CHILE (2011) Comunidad Indígena de la Isla Traiguén espera nulidad de venta de terreno efectuada por el Ejército, disponible en: http://www.biobiochile.cl/2011/04/12/ comunidad-indigena-de-la-isla-traiguen-espera-nulidad-deventa-del-terreno-efectuada-por-el-ejercito.shtml, revisado en septiembre 2015.

BONNAL, P., BOSC P., DÍAZ M. y LOSCH, B. (2004) "Multifuncionalidad de la agricultura y nueva ruralidad: ¿reestructuración de las políticas públicas a la hora de la globalización?, En: Edelmira Pérez y Adelaida Farah (Comps.), Desarrollo rural y nueva ruralidad en América Latina y la Unión Europea, Bogotá: Pontificia Universidad Javeriana, pp. 19-41.

CARTON DE GRAMMONT, H. (1999) Agricultura de exportación en tiempos de globalización, México: Juan Pablos Editor.

CARTON DE GRAMMONT, H. y MARTíNEZ, L. (2009) La pluriactividad en el campo latinoamericano, Quito: Facultad de Ciencias Sociales.

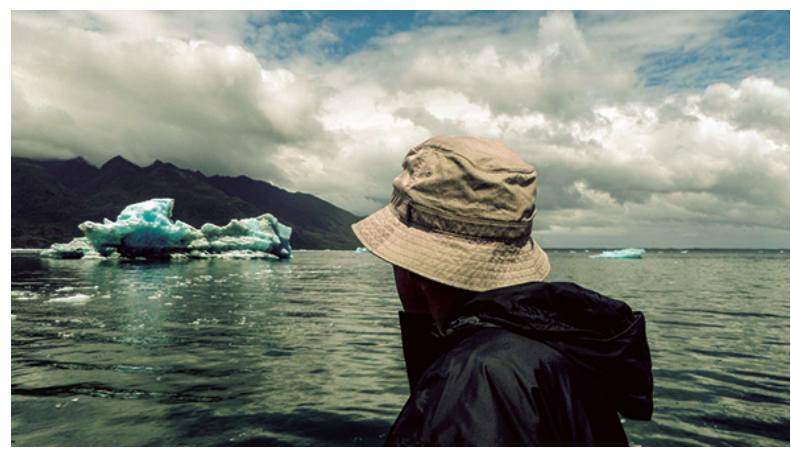


CRAVIOTTI, C. (2013) La agricultura familiar en Argentina: ¿Fortalecimiento institucional y debilitamiento estructural?, XXIX Congreso de la Asociación Latinoamericana de Sociología.

DIARIO AYSÉN (2015a) Alto Mañihuales: empresa minera, autoridades, y comunidad; enfrentados por la contaminación ambiental, disponible en: http://diarioaysen.cl/ regional/alto-manihuales-empresa-minera-autoridades-ycomunidad-enfrentados-por-la-contaminacion-ambiental/, revisado en septiembre 2015 .

DIARIO AYSÉN (2015b) Contaminación en Alto Mañihuales: Siete pobladores arrojaron positivo por presencia de arsénico y aseguran que autoridades no han informado de riesgos futuros, disponible en: http://www.diarioaysen. cl/sitio/2015/11/20/contaminacion-en-alto-manihualessiete-pobladores-arrojaron-positivo-por-presencia-dearsenico-y-aseguran-que-autoridades-no-han-informado-deriesgos-futuros/,revisado en diciembre 2015.

DIMITRIU, A. (2002) Producir y consumir lugares: Reflexiones sobre la Patagonia como mercancía, versión revisada del trabajo presentado en Jornadas Transformaciones Sociales y Reestructuración Capitalista del Siglo XX (Dimensiones económicas, sociopolíticas y espaciales), organizado por el Programa de Historia de las Relaciones Sociales entre Estado, Economía y Sociedad, CEI/UNQ, Universidad Nacional de Quilmes, 24 de agosto de 2001.

FAO (2014) ¿Qué es la agricultura familiar? Disponible en http:// www.fao.org/family-farming-2014/home/what-is-familyfarming/es/, revisado en marzo 2015.

GOBIERNO REGIONAL DE AYSÉN \& CEPAL (2009) Estrategia Regional de Desarrollo de Aysén, Santiago.

GOBIERNO REGIONAL DE AYSÉN (2011) Plan Regional De Ordenamiento Territorial: Región de Aysén, Coyhaique.

GÓMEZ, S. (2000) ¿Nueva Ruralidad? Un aporte al debate, trabajo presentado en Seminario Internacional: La Nueva Ruralidad en América Latina, Pontificia Universidad Javeriana. Facultad de Estudios Ambientales y Rurales, Maestría en Desarrollo Rural, Bogotá, 22 - 24 de Agosto 2000.

HAESBAERT, R., (2004), O Mito da Desterritorialização, Rio de Janeiro: Bertrand Brasil.

HARTMANN, P. (2009) Región de Aisén - Chile: dos décadas de ordenamiento territorial y propuestas de estrategias y modelos de desarrollo sustentable, en Revista de Urbanismo $\mathrm{N}^{\circ} 20$, junio, Santiago de Chile.

HARVEY, D. (2001) Spaces of Capital towards a Critical Geography, New York: Routledge.

HARVEY, D. (2014) Diecisiete contradicciones y el fin del capitalismo, Quito: Editorial IAEN.

LANGMAN, J. \& MOORE, N. (2013) Saving Futaleufú, the big river under threat, en Patagon Journal, $\mathrm{N}^{\circ} 4$.

MASALLERAS, M., \& ORTEGA, R. (2012) Fronteras interiores; Una contribución del ejército vigente, Memorial del Ejército de Chile (488), Santiago de Chile: (D. C. Ejército, Ed.), pp. 135-147.
MOSS, L. (2006) Migración de amenidad: búsqueda y preservación de las montañas y sus culturas, Nuevo México: Ed. L.A.G. Moss.

NÚÑEZ, A., ALISTE, E. y BELLO, A. (2014) El discurso del desarrollo en Patagonia-Aysén: la conservación y la protección de la naturaleza como dispositivos de una renovada colonización, ponencia en el XIII Coloquio Internacional de Geocrítica, El control del espacio y los espacios de control, Barcelona, 5-10 de mayo.

PERIÓDICO LA SEGUNDA (2013) Fiebre del Oro en Aysén, Mineras ya controlan 635 mil hectáreas, disponible en: http://www. lasegunda.com/Noticias/Economia/2013/06/856053/ fiebre-del-oro-en-aysen-mineras-ya-controlan-635-mil-hectareas, revisado en diciembre 2014.

PERIÓDICO LA TERCERA (2011a) Negocio de los lodges se triplica en los últimos 10 años en la Patagonia, disponible en: http://diario.latercera.com/2011/02/20/01/contenido/ negocios/27-59834-9-negocio-de-los-lodges-se-triplica-enlos-ultimos-10-anos-en-la-patagonia.shtml.

PERIÓDICO LA TERCERA (2011b) ¿Cómo avanza el mercado de los bonos de carbono en Chile?, disponible en reportaje del diario La Tercera, en: http://diario.latercera. com/2011/06/25/01/contenido/tendencias/2673991-9-como-avanza-el-mercado-de-los-bonosde-carbono-en-chile.shtml_revisado en septiembre 2015.

REYES. S. y RODRÍGUEZ, J. C. (2015) Proyecto Hidroaysén: capitalismo extractivista, regulación estatal y acción colectiva en la Patagonia, en Revista Polis [En línea], 40 | 2015, publicado el 17 mayo 2015, consultado el 22 diciembre 2015, http://polis.revues.org/10819.

RODRÍGUEZ, J. C., GISSI, N., THER, F. \& MEDINA, P. (2012) La Patagonia en escena: Auge minero, demanda energética y geopolítica del neoliberalismo, trabajo presentado en el Congreso Chileno de Sociología 2012, Pucón, disponible en: http://sociologiadesarrollo.wordpress.com/2012/ 10/30/7-congreso-chileno-de-sociologia-pucon-2012/, revisado agosto, 2013.

RODRÍGUEZ, J. C., MEDINA, P. y REYES, S. (2014) Territorio, Paisaje y Marketing Global. Imaginarios en la construcción de la Patagonia como marca, Magallania, [s.l.], v. 42, $\mathrm{N}^{\circ} 2$, oct, disponible en: http://www.institutodelapatagonia.cl/index. $\mathrm{php} / \mathrm{magallania/article/view/623/592}$, revisado en junio 2015, pp. 109-123.

RODRÍGUEZ, J. C., GISSI, N. y MEDINA, P. (2015) Lo que queda de Chile: La Patagonia, el nuevo espacio sacrificable, Revista Andamios, v.12, $\mathrm{N}^{\circ}$ 27, enero-abril, pp. 335-356.

ROMERO, H. (2009) Comodificación, exclusión y falta de justicia ambiental. Globalización y territorio en América Latina, VIDIO, Delgado Mahecha y Hellen Cristancho Garrido, Editores, Biblioteca Abierta, Colección General, serie Geografía, Universidad Nacional de Colombia, Facultad de Ciencias Humanas, Departamento de Geografía, pp. 410-453.

SAAVEDRA, G. (2007) Las economías silenciosas del litoral aisenino, en Osorio, M., Saavedra, G., y Velásquez, H., "Otras narrativas en Patagonia: tres miradas antropológicas a la Región de Aysén", Santiago de Chile: Ed. Ñire Negro. 
SÁENZ, J. (2015) "Territorio rural y sus transformaciones ante procesos de globalización en la subregión transandina de la Provincia de Palena, Patagonia chilena", Memoria para optar al título de Antropólogo, Departamento Antropología, Universidad de Chile.

SALAS, H., RIVERMAR, L. y Velasco P. (2011) Nuevas Ruralidades. Expresiones de la transformación rural en México, México, D. F.: UNAM-IIA-Juan Pablos Editor.

SANTOS, M. (2000) La naturaleza del espacio. Técnica y tiempo. Razón y emoción, Barcelona: Ariel.

SERJE, M. (2011) El revés de la Nación: Territorios salvajes, fronteras y tierras de nadie, Colombia: Ediciones Uniandes.

SERNAPESCA (2015) Boletín Sectorial $N^{\circ}$ 1, Sernapesca Aysén.

VILLAGRÁN, J., NÚÑEZ, A. e HIDALGO, R. (1997) "Políticas públicas y Ocupación del Territorio en la XI Región de Aysén", en Revista de Geografía Norte Grande, $N^{\circ} 24$, Santiago de Chile, Instituto de Geografía de la PUCCH.

\section{NOTAS}

1 Se han identificado por lo menos nueve ecorregiones en la Patagonia occidental-Aysén (Silva en Hepp y Neal, 2014), desde las zonas templadas con pluviometrías de $2500 \mathrm{~mm}$ anuales en el litoral, al reino nevado de tundra, glaciales y nieves sobre los 1200 msnm, pasando por la seca estepa fría y los bosques andinos boreales.

2 Caso registrado en Alto Palena, 2014.

3 Alto Mañihuales: empresa minera, autoridades, y comunidad; enfrentados por la contaminación ambiental. Disponible en Diario Aysén: http://diarioaysen.cl/regional/alto-manihuales-empresaminera-autoridades-y-comunidad-enfrentados-por-la-contaminacion-ambiental/, recuperado en Septiembre, 2015.

4 Disponible en http://www.diarioaysen.cl/sitio/2015/11/20/ contaminacion-en-alto-manihuales-siete-pobladores-arrojaronpositivo-por-presencia-de-arsenico-y-aseguran-que-autoridadesno-han-informado-de-riesgos-futuros/, recuperado en diciembre de 2015.

5 Reportaje del periódico nacional La Segunda, disponible en http://www.lasegunda.com/Noticias/Economia/2013/06/856 053/fiebre-del-oro-en-aysen-mineras-ya-controlan-635-mil-hectareas, recuperado en diciembre, 2014.

6 Localidad aislada de Futaleufú con áreas que fueron solicitadas para la exploración y explotación minera.

7 Todos los datos son extraídos del Boletín sectorial N 1-2015 de SERNAPESCA-Aysén.

8 Reportaje La Tercera, Negocio de los lodges se triplica en los últimos 10 años en la Patagonia, disponible en: http://diario.latercera.com/2011/02/20/01/contenido/negocios/27-598349-negocio-de-los-lodges-se-triplica-en-los-ultimos-10-anos-en-lapatagonia.shtml.

9 Disponible en reportaje del diario La Tercera, en: http://diario. latercera.com/2011/06/25/01/contenido/tendencias/26-739 91-9-como-avanza-el-mercado-de-los-bonos-de-carbono-en-chile.shtml, revisado al 15 de septiembre de 2015.
10 Disponible en reportaje de Revista Qué Pasa en: http://www. quepasa.cl/articulo/negocios/2012/03/16-7938-9-el-otro-acuerdo-en-aysen.shtml/, revisado al 15 de septiembre de 2015.

11 Disponible en Radio Bíobío en: http://www.biobiochile.cl/2011 /04/12/comunidad-indigena-de-la-isla-traiguen-espera-nulidad-de-venta-del-terreno-efectuada-por-el-ejercito.shtml, revisado el 15 de septiembre de 2015. 